\title{
Investigation of Cognition with Schema Model
}

\author{
Gefei $\mathrm{Mu}^{1}$ \\ ${ }^{1}$ Dubai America Academy, 00000 Hessa Street - Sheikh Zayed Rd, Dubai, United Arab Emirates
}

\begin{abstract}
Schema model (schema network) is a model established on the concept of neural network, networking memory, and the subconsciousness. It is also related to psychoanalysis which is barely seen in papers of neuroscience today. This paper reviews different pre-existing theories around "cognition" and schema. To some extent, it redefines subconscious without being overly philosophic and creates a system which is another perspective to view cognition. The schema model is composed of three parts, including input, subconsciousness and cognition and it is observed that cognition can only do three things, which are reasoning, imagination and centre executive. The subconsciousness is composed of some well associated models and unassociated models, as people can conclude the associated models from environment but unassociated models usually cannot be obtained as they are learnt because of the gatekeeper. The original schema theory explains all the behaviours [8] but predicts little things, so the author proposes a schema network by combining the theory with interdisciplinary knowledge to explain human behaviours more thoroughly. The purpose is to explain cognition, subconsciousness of human in a new way, and hopefully people can use it to find implication on education, sociology and artificial intelligence, or prevention of mental disorders.
\end{abstract}

\section{Introduction}

Human brains conclude pattern from RLE, "Real Life examples" (input and output). The pattern is called model (or knowledge) and human use the pattern that the brains have learned to predict the output when new input comes [5]. If no one ever taught people what to do, they would learn directly from nature as they grow up. However, the knowledge is likely to become provincial because they are concluded in only one circumstance. Although they might be provincial, people believe in them deeply because the models are supported by RLE. Their emotions, predictions, attitudes are aroused as they using these models. Also, these models actually are so sedentary that they get aroused by stimulus over and over again and predict the environment "successfully" [9].

As these models have strong support of the real examples, brains will arouse it over those unassociated models that the gatekeepers have taught them. That makes personality hard to change through CBT or other well-known cognitive treatment because CBT does not stop people from arousing negative models that people concluded, but it adds more positive model that arouse align the pre-existing negative models. Moreover, these positive models are likely to be unassociated models because the CBT does not give people different environments to learn associated model. The findings are not to criticize the CBT but to explore the principle of consciousness, and subconsciousness that possibly explain why cognitive treatment do not solve the problems.

\section{Only Workable Model}

If one person only has limited and failure examples, it is natural and inevitable for this individual to conclude an only workable model. Most of the relative behaviours or cognition is bound to the only workable model. Doctors may persuade one that this only workable model is limited and provincial and not helpful at all [9]. But the positive model will not be "strong" enough to influence the arousal of models of subconsciousness. That is because the positive model lacks the vivid examples to support it and as people are in the situation, the external stimulus naturally does not arouse the targeted positive model. The subconsciousness is still arousing negative models. It is well observed that patients' conditions will be improved for intake of the positive models at the middle of the treatment. But as the treatment stops, the positive models' influence start to diminish. That diminishing effect could be the result of the internal arousal chain (See input).

As the only workable models pile up, they then become less adaptive schema-individuals with less adaptive schema will choose to leave the environment, but if they cannot, these schemas eventually become dysfunction beliefs. There are two possible ways to solve the problem. The first one is to experience a real situation in which the people can conclude that the pre-existing model do not work as they are using the models. The other way is to experience more situations, until the brain concluded the overarching model beyond their only workable model. In modern society, every task 
has some wired similarity, one cannot or does not need to acquire examples from different environments. It makes people have low cognitive flexibility. And one way to effectively prevent different mental disorder is to revolutionize the current social system which enable and encourage people to experience more environment.

\section{Analysis}

\subsection{Schema model}

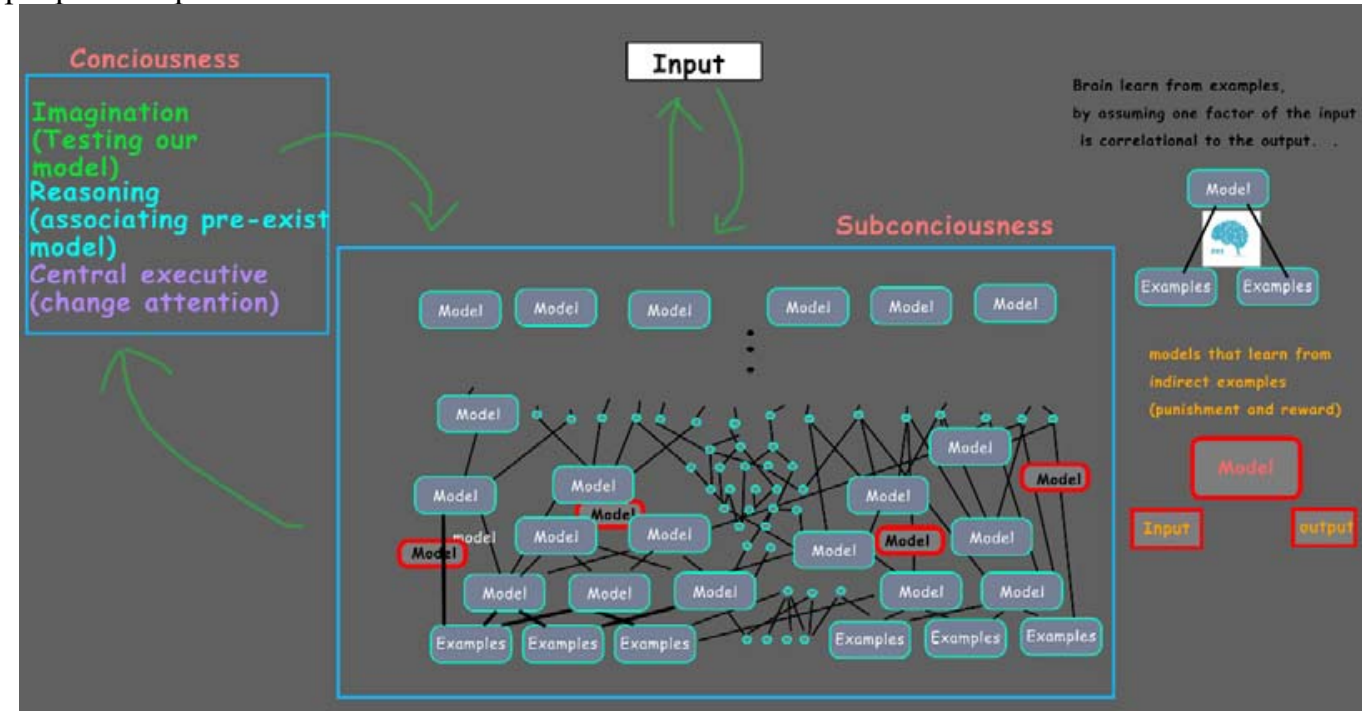

Fig. 1. Schema model

In the subconsciousness, models in blue means the associated model, and models in red means the unassociated model. Those blue nodes are the simplified form of blue model; straight black lines represent links and association. The Input refers to both internal input and external inputs. External inputs are the environment stimulus that we perceive, and the internal input means the inner stimulus that we give to ourselves and they form an internal model chain as we ruminate. The consciousness is believed to function beside the subconsciousness, it does not directly link to input. The examples at the bottoms of the subconsciousness are the flashbulb memory. Each of the example contains a complete input/output relationship. At the beginning, people concluded model from examples (flashbulb memory), and then later they might start to conclude models from models. The models can be procedural or episodic, and there should be a lot of models. The question is how the brain concludes them and how our brains learn these patterns $[5,6]$.

\subsection{Subconsciousness [7]}

The networks of models which is shown in figure 1 is arbitrarily called subconsciousness. Psychoanalysis might have different definitions of subconsciousness. However, subconsciousness in this paper can be referred to the networks of models that wait to be aroused $[2,3]$. Models are called knowledge units. Associated models, which are in blue, are connected with examples. It means that those models have convincing memory (examples) to support it [5]. Unassociated models, which are in red, do not have convincing memory to support it. These unassociated models are created through punishment and rewards, models that individuals are unlikely to learn in the environment. If gatekeepers force them to accept this model, people have higher chance to learn the wrong relationship of the input/output from environment. These two types of models sometimes conflict each other, resulting in inefficient and conflicting schema, weakening people's adaptive power, and it is believed that those conflicts result eventually in negative schema which later cause depression. It was not the belief that he will failed, but the conflict between the belief that predicts he will fail and the belief that predicts that he must do.

\subsection{Consciousness}

The term "consciousness" in this paper means the most likely cognition and thinking process, because some researches have shown that the even with absence of consciousness, different areas of the brains still can be activated based on the types of stimulus they perceive. When people are in paralysis, they can still react to the environment without consciously aware of it [3]. Although the researches study mainly on those whose eyes are closed that cannot react to visual information, it is reasonable to assume that in terms of visual information, it is not necessary to go through consciousness too as they are solved by subconsciousness. Therefore, re-consider the meaning of terminology is very important. It is thought that cognition has only three functions: reasoning, imagination [11] and central executive [8]. And all of them are defined differently from what they have been widely known.

Reasoning refers to the process by which creatures combine different pre-existing models to form a new model and put the new model back in to our 
subconsciousness in order to solve the input at the next cycle. This reasoning process is not rewarded by emotions. One factor that triggers the reasoning process is that when people's subconsciousness cannot directly solve the input, which means that they cannot directly be scanned in subconsciousness.

Central executive controls our attentions, one rule that has been observed to change our attention is surprise. However, researches do not fully explain what trigger surprise. This paper argued that attention is mainly determined by these unsolvable inputs. When people perceive things (even though they do not know they are perceiving them) that cannot be directly solved by the pre-existing models in the subconsciousness, central executive will send correlated models or memory into consciousness for reasoning to combine. This process is the attention and the change of attention always includes indirectly input and correlated models or correlated memory. Reasoning and attention are very similar. If every input in the environment can be directly solved by subconsciousness, people might stop thinking. Boredom might be the result of too much associated model. However, it is unlikely that people will stop thinking because human can keep their internal input chains much longer than animals.

Imagination means the process by which brains use pre-existing examples to test the models, either models that are created in the reasoning, or models that already in subconsciousness. People can use their memories (example of input/output) to test if their models work under some situations, or use their pre-existing model to test the output from an input. One thing that we want postulated is that if the models have been tested by our imagination, or reality. Then the "proud "chemical might be secreted to buff the neuron pathways to enhance the model, which might be supported by the modern research of neuron networks.

In the schema networks, it is believed that cognition is established on the subconsciousness. All of the cognitive activity relies on different combination of pre-existing models that are aroused by stimulus. The neuron network in human brains might be similar to hard disks. If people do not perceive information, they still have grey matter and neuron networks, but these networks of neurons do not carry information. The subconsciousness is empty as people are first born.
However, because of the function of consciousness and the emotions, brains start to memorize examples because of chemical of fear, and combine models from these examples and eventually form a network in the subconsciousness to make people make sense of the world. One imagined research to examine the theory is to have a 30 years old male who have dormant since his born, and measure the synaptic pruning in his brains while he is interacting with environment.

\subsection{Input}

The input that appear at the top of the schema network represent the both external environment we perceive and the internal input chain. In the internal input chain, Central executive will "send" models from the subconsciousness to the consciousness based on if the subconsciousness can directly scan the models. If the subconsciousness cannot directly solve the input, then it will start to send correlated models and memory to the consciousness. As these models go through the" combining and testing phrase". A new model might be generated and this new model can interrupt the original process. In this case, brains might no longer keep on what the external inputs were, but they get interrupted repeatedly by the new internal inputs and model fetched in the subconsciousness continue to arouse more models. Furthermore, modern researches have indicated that when people have a special composition of genes which determine the attributes of their body, including their working memory, sensation, attention et cetera, they might be more prone to different mental disorders [10]. The schema model is supported by the finding, the speed of transferring, capacity, and sensitivity is different among individuals and the conjecture is that, if two individuals have same genetic compositions and have exactly the same subconsciousness, then these two persons should react to the environment perfectly identical.

\subsection{Processing}

Figure 2 briefly illustrates the overall process of how schema model works. 


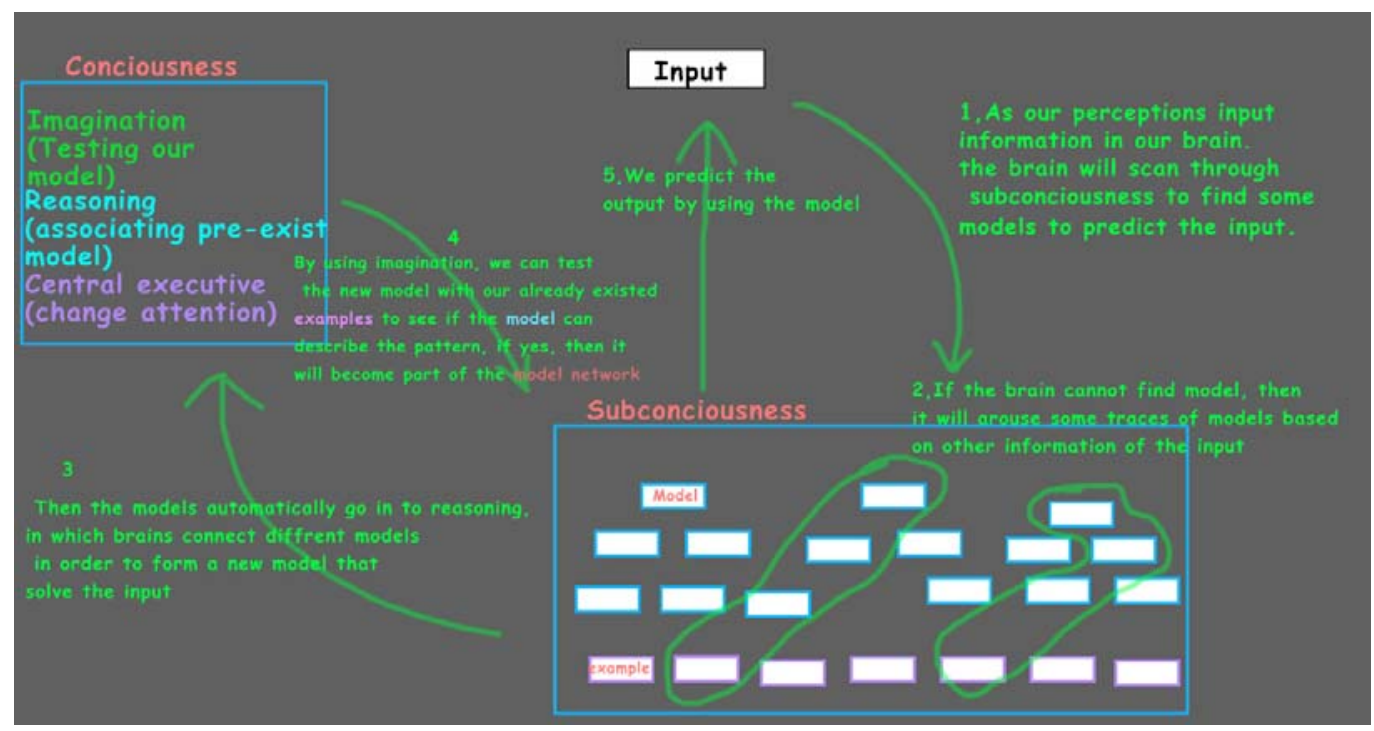

Fig. 2. The procedure

Step 0, perceive external input.

Step 1, all the inputs people perceive will go to the subconsciousness. For those inputs that they can directly solved by the model's networks, people will not be aware of them, but they will make sense. For those inputs that are not directly solvable, central executive will send some correlated models that are from different parts of the brain into the consciousness for the reasoning and imagination process to run.

Step 2, reasoning use these correlated models to form new model that has the potential to solve the issue. Imagination then tests the new model by fetching additional knowledge from the subconsciousness, this process might interrupt the former cycle and lead to a new literation.

Step 3, after the new model has been tested, the brain will put the new model back to a place of the subconsciousness and use the new model to predict the output at the next cycle. If the new model successfully predicts the output in reality, then the brain might store it as an associated model for a bit longer. And the learning condition (input, output) becomes the memory to trigger it.

Next time, when the situation similar to the memory happen, the associated model would be aroused, the neuron pathway gets buffed. An example is that how people recognize objects like door. At the beginning, we do not know what door is, so our brains should have used fear to remember the example for it, but as we see more and more doors, the brains start to compare these memories about doors, therefore the comparison is triggered. At the second time when we see them, it is common to have the correlated memory that is enough to arouse attention. These memories will start to be combined in the reasoning, then once the model has been tested in our imagination, we will feel proud and "proud chemical" makes the brains remember the model for a while. If it solves the problem in reality, and then a large amount of proud chemical will be released and it will be stored in our subconsciousness for much longer time.

\section{About Emotion $[1,10]$}

Some people argued that emotions are the combination of different levels of arousal and pleasantness [1]. This way of categorizing emotion may be effective in explaining the cultural content. However, this method of categorisation is ethnocentric and do not actually tells why human have emotions [1]. Before the people have enough models to make sense of the world, when their subconsciousness is empty what gives them the task? And who can tell them what model should be learned, and what models should be discarded [7]? In Modality, the emotion is believed to be the biggest reward system that play a fundamental role of modelling.

Emotions make the modelling process to have flex adaption. Many emotions like happy, proud, frustrated can be categorized as the rule of modelling. It seems that proud is triggered after our model have been tested in reality; frustration is the result of our model that once work does not work for internal reason. Anger is triggered when the model that once works does not work for external reason. Sadness is triggered the failure of doing what their model describes, and fear is triggered when the model cannot solve the input. Biologically, it has been observed that different emotions may actually use same type of chemical but in different amount. Their findings can indirectly support the schema model that emotions are correlated to the modelling [10]. There are some emotions specials. For instance, love and disgust. Love and disgust might be the evolutional "pre-built" emotions that are not influenced by the schema network learned of the individuals.

\section{Conclusion}

The modality creates a model called schema model in an attempt to redefine the function of consciousness, subconsciousness, and emotion. The emotions are the biggest reward system in creatures, determining what lives should remember in order to predict the 
environment. The consciousness is considered as a support to increase the learning speed tremendously, and for subconsciousness to learn models more quickly. The strength of the schema model is that it links most of the presented psychologic theory into the process of the modelling. However, one disadvantage of the schema model is that it is only a conjecture which has no empirical evidence to support it, meaning it is prone to making errors, even mistakes. Yet the author still hopes that this paper can provide some insightful ideas to those who are interested in it. Basically, the unassociated model and associated model can be used to predict the vulnerability of depression of a person, and it might be another way to view individuals and its relationship with society and environment.

\section{Acknowledgement}

Thanks to professor Jeff Dalley and Dr. Zhou who advise and review the theories of the paper. And provide modern epigenetic explanation of behaviour. Thanks to Dr. Yjango' insightful theories of learning and lives evolution. Appreciate Sigmund Freud, carl Jung, Alter, anna Freud et. al's legacy of lives - the knowledge of the psychoanalysis.

\section{References}

1. Juan Carlos Ortiz Nicolás, Marco Aurisicchio, Pieter Desmet, Pleasantness and arousal in twenty-five positive emotions elicited by durable products, 9th International Conference on Design and Emotion 2014: The Colors of Care Project: Design and Emotions, October 2014, DOI: 10.5281/zenodo.2605626.

2. Steven Novella, Reporting Medical Cases as Human Interest Stories: Chase Britton Edition, Neuroscience, Science and the Media Comments, February 2011, accessed on November 10th, 2020, retrieved from.https://theness.com/neurologicablog/index.php/ reporting-medical-cases-as-human-interest-stories-c hase-britton-edition/.

3. Sue McGreevey, Finding signs of life when it matters most, FMRI on Unconsciousness patient, July 2017, accessed on November 10th, 2020, retrieved from https://news.harvard.edu/gazette/story/2017/07/using -fmri-eeg-to-search-for-consciousness-in-icu-patient $\mathrm{s} /$.

4. Diane Martindale, October 2005, Identifying faces, accessed on November 10th, 2020, retrieved from https://www.scientificamerican.com/article/one-face -one-neuron/

5. Yjango, How do human brains learning, June 2020, accessed on November 10th, 2020, retrieved from https://zhuanlan.zhihu.com/p/27857399

6. Hebbian, The Organization of Behavior: A Neuropsychological Theory 1949, accessed on November 10th, 2020 from https://en.wikipedia.org/wiki/Hebbian_theory

7. Convolutional neuron network. Deeplizard, Convolutional Neural Networks (CNNs) explained Dec 10, 2017, accessed on November 10th, 2020 from https://www.youtube.com/watch?v=YRhxdVk_sIs

8. Ballet theory: Brewer, William F. Bartlett's concept of the schema and its impact on theories of knowledge representation in contemporary cognitive psychology, accessed on November 10th, 2020 From https://psycnet.apa.org/record/2000-07376-005

9. CBT: peter trower Cognitive Behavioural Counselling in Action Third Edition nov 2015, accessed on November 10th, 2020 from library...

10. The endophenotype concept in psychiatry: UC San Diego - Department of Psychiatry,May 2 2019, accessed on November 10th, 2020 from https://www.youtube.com/watch?v=3q2QoDngqJI

11. Theory of knowledge, IB diploma 3rd edition, 2020 accessed on November 10th, 2020 from library 